\section{More iPS cells in less time}

\section{By Kai-Jye Lou, Staff Writer}

A team at The Scripps Research Institute has found that a trio of small molecules can provide more than a 200 -fold boost in the generation of induced pluripotent stem cells from human fibroblasts while cutting the generation time in half. ${ }^{1}$

Fate Therapeutics Inc. has exclusively licensed the associated technology. Coupled with IP for an earlier fibroblast reprogramming protocol that only uses recombinant proteins, ${ }^{2}$ which the biotech also obtained from Scripps, the company hopes to use the technologies to generate pharmaceutical-grade induced pluripotent stem (iPS) cells for therapeutic use and drug discovery on an industrial scale.

Before iPS cells could be used for either application, "we needed to find a way to 'industrialize' the technology to quickly and efficiently create iPS cells," said Fate Therapeutics CTO Dan Shoemaker.

The first step was to get rid of viral vectors and DNA plasmids, which are the conventional vectors used to deliver the transcription factors needed to reprogram somatic cells into iPS cells. These vectors raise safety concerns because the viral or DNA genetic sequences can integrate into the cellular genome, which can cause unwanted side effects.

The next step was to optimize the efficiency and speed of iPS cell generation.

Enter Sheng Ding, an associate professor in the Department of Chemistry at Scripps and a scientific cofounder at Fate Therapeutics. In April, Ding provided a solution to the vector issue with a protocol that only used recombinant transcription factor proteins to reprogram fibroblasts into iPS cells ${ }^{2}$.

Ding's latest publication addressed the efficiency and speed issue.

Indeed, the field still relies on genetic methods such as viral and plasmid vectors to get good efficiencies, according to Paul Knoepfler, an assistant professor in the Department of Cell Biology and Human Anatomy at the University of California, Davis. "While nongenetic approaches have been formally proven to work, the efficiencies are so low that practically speaking it remains unclear how helpful those technologies will be on their own," he said.

"The low efficiency and slow speed of reprogramming, which had a success rate of roughly 1 in 10,000 cells and took about 4 weeks from start to finish, presented a potentially greater problem for the ultimate applications of human iPS cells," said Shoemaker.
Low efficiency and protracted generation times can result in a heterogeneous cell population that includes nonreprogrammed cells and cells that have acquired undefined genetic and epigenetic abnormalities.

"The reprogramming process is stressful to the cells as oncogenes are activated and tumor suppressors are suppressed," Ding told SciBX. "The longer cells remain in culture, the more likely they are to accumulate undefined genetic and epigenetic alterations. This could cause the cells to behave unpredictably and render them unsuitable for therapeutic and disease modeling applications."

To address this issue, the Scripps team set out to find small molecule modulators of pathways involved in reprogramming processes or cell survival. They identified a trio of small molecules that improved the efficiency and reduced the time required to generate iPS cells from human fibroblasts being reprogrammed with viral vectors.

The compounds were SB431542, an inhibitor of transforming growth factor- $\beta$ receptor 1 (TGFBR1; ALK5); PD0325901, which blocks mitogen-activated protein kinase kinase (MEK); and thiazovivin, a compound that improves cell survival via an undisclosed mechanism.

Although the researchers still used viral vec-Dan Shoemaker, tors to reprogram the fibroblasts, Ding expects that the small molecule cocktail could also be combined with the protein-based reprogramming protocol that his group published in April.

The results were published in Nature Methods.

"With this method, researchers could produce iPS cells with greater consistency and in greater numbers," said Ding, the paper's senior author.

"This study shows that with 3 chemicals we can create 200 times more cells in half the time as the regular methods," Shoemaker told SciBX. "We expect that these new chemicals will easily translate and can immediately be applied to any reprogramming method."

In particular, he said the use of the three small molecules in combination with Fate Therapeutics' previously licensed protein-based reprogramming method could "offer a highly efficient, nonviral, nonDNA-based process of reprogramming" and "an opportunity to recapitulate human physiology for drug discovery and therapeutic use."

"It is much easer to develop disease models with iPS cells than with embryonic stem cells," noted Leonard Zon, a scientific cofounder of Fate Therapeutics who is director of the stem cell research program at Children's Hospital Boston at Harvard Medical School and an investigator at the Howard Hughes Medical Institute.

Zon thinks Fate Therapeutics' technologies could promote the use of iPS cells in drug discovery and screening. "I think industry is very interested in turning iPS cells into liver cells so companies could test to see if compounds are being metabolized," he told SciBX.

Zon added that the approach could make it practical to expose human tissues derived from iPS cells to libraries of compounds to reproduce disease symptoms and then screen for compounds that can reverse such symptoms. 
However, Knoepfler at UC Davis noted that the safety profile of iPS cells generated with nonviral, non-DNA methods still needs to be fleshed out.

"We all have long assumed nongenetic approaches would yield safer iPS cells, but if there is any data out there from testing that hypothesis, it has not been published," he said. "This leaves us with the key question facing the field-will iPS cells make it to the clinic as we all hope, or will they mainly be used in vitro for modeling disease states?"

Ding's group is now looking for new small molecules that could further improve the efficiency of reprogramming human cells. "We want to keep identifying small molecules that could be better combinations," he said.

Eventually, Ding also wants to take protein-based reprogramming agents out of the equation and replace them with small molecules. "Ultimately, we want to move toward a protocol that only uses small molecules to efficiently reprogram fibroblasts into iPS cells," he told SciBX.

The desire to move away from proteins is motivated by cost. "Proteins are expensive but are otherwise fine as reprogramming agents," said Ding.

Scripps has filed multiple patents covering the use of proteins and small molecules to generate iPS cells. The discoveries reported in Nature Methods and the protein-based reprogramming approach published earlier this year were both sponsored by Fate Therapeutics.

Lou, K.-J. SciBX 2(42); doi:10.1038/scibx.2009.1557

Published online Oct. 29, 2009

\section{REFERENCES}

1. Lin, T. et al. Nat. Methods; published online Oct. 18,2009 ; doi:10.1038/nmeth.1393

Contact: Sheng Ding, The Scripps Research Institute, La Jolla, Calif. e-mail: sding@scripps.edu

2. Zhou, H. et al. Cell Stem Cell 4, 381-384 (2009)

COMPANIES AND INSTITUTIONS MENTIONED

Children's Hospital Boston, Boston, Mass.

Fate Therapeutics Inc., La Jolla, Calif.

Harvard Medical School, Boston, Mass.

Howard Hughes Medical Institute, Chevy Chase, Md.

The Scripps Research Institute, La Jolla, Calif.

University of California, Davis, Calif. 